\title{
Inadequate iodine intake is associated with subfecundity in mild-to-moderately iodine deficient Norwegian women
}

\author{
Anne Lise Brantsaeter $^{1}$, Marianne H. Abel ${ }^{1,2}$, Ida H. Caspersen ${ }^{1}$, Verena Sengpiel ${ }^{3,4}$, \\ Bo Jacobsson $^{3,4}$, Per M. Magnus ${ }^{1}$, Jan Alexander ${ }^{1}$ and Helle Margrete Meltzer ${ }^{1}$ \\ ${ }^{1}$ Norwegian Institute of Public Health, Oslo, Norway, \\ ${ }^{2}$ TINE SA, Oslo, Norway, \\ ${ }^{3}$ Sahlgrenska University Hospital, Gothenburg, Sweden and \\ ${ }^{4}$ University of Gothenburg, Gothenburg, Sweden
}

\section{Abstract}

Iodine is an essential micronutrient and an integral part of the thyroid hormones. In women of childbearing age, the estimated average iodine requirement is $95 \mu \mathrm{g} /$ day and the recommended daily intake is $150 \mu \mathrm{g} / \mathrm{day}$. While severe iodine deficiency poses reproductive risks, including infertility and abortions, the potential impact of mild-to-moderate iodine deficiency on subfecundity is unknown.

We examined whether iodine intake was associated with risk of subfecundity (i.e. $>12$ months trying to get pregnant) in a large cohort of mild-to-moderately iodine deficient women.

Women enrolled in the Norwegian Mother and Child Cohort Study in gestational week 15 were asked to report whether the pregnancy was planned and how many months the couple had sexual relations without any contraception before getting pregnant. Information about time to pregnancy, maternal characteristics and iodine intake was available for 56,416 planned pregnancies. The median (interquartile range) time to pregnancy was $1.5(0.5-6.0)$ months and the prevalence of subfecundity was $10.8 \%)$. We used iodine intake assessed by a validated food frequency questionnaire administered in pregnancy as a proxy for long-term (prepregnancy) iodine intake. We used logistic regression to estimate the association between iodine intake and subfecundity, using flexible modelling with restricted cubic splines, and adjusted for maternal age, BMI, parity, education, smoking status, energy intake and fiber intake. The median calculated iodine intake was $121 \mu \mathrm{g} /$ day and the median urinary iodine concentration in a subsample of $\mathrm{n}=2795$ women was $69 \mu \mathrm{g} / \mathrm{L}$.

The prevalence of subfecundity was lowest for iodine intakes $\sim 100 \mu \mathrm{g} / \mathrm{day}$ and increased at lower intakes $(\mathrm{p}$ overall $=0.005)$. Compared to an intake of $100 \mu \mathrm{g} /$ day (reference), intakes $\sim 75 \mu \mathrm{g} /$ day was associated with $5 \%(95 \% \mathrm{CI}$ : 1\%, 9\%) higher prevalence and intakes $\sim 50 \mu \mathrm{g} /$ day with $14 \%(95 \% \mathrm{CI}: 4 \%, 26 \%)$ higher prevalence. Use of dietary supplements was recorded only for the last 6 months prior to conception and women were included in the analysis regardless of their reported supplement use. In a sensitivity analysis, we excluding women who reported iodine-containing supplement use in the period 26-9 weeks before conception and the result remained unchanged. We also modelled time to pregnancy by Cox regression, and the result was consistent with the result for subfecundity.

The only good dietary sources of iodine in Norway are milk and white fish, and many women of fertile age have low intakes of these food items. This study shows that low habitual iodine intake may be a risk factor for subfecundity.

\section{Conflict of Interest}

Marianne H. Abel is employed by a Norwegian dairy company (TINE SA), and she participates in this project as an industrial Ph. D.-student financed partly by the dairy company and partly by The Research Council of Norway. This project is designed, owned and administered by The Norwegian Institute of Public Health and analysis of the data follow from protocol. All results of analysis in the project are to be published regardless of the results. The other authors have no conflicts of interest. 